\title{
Novas combinações em Atanus Oman (Hemiptera, Cicadellidae) ${ }^{1}$
}

\author{
Keti Maria Rocha Zanol ${ }^{2}$
}

${ }^{1}$ Contribuição número 1560 do Departamento de Zoologia, Universidade Federal do Paraná.

${ }^{2}$ Departamento de Zoologia, Universidade Federal do Paraná, Caixa Postal 19020, 82531-980 Curitiba-PR, Brasil. kzanol@ufpr.br.

\begin{abstract}
New combinations within Atanus Oman (Hemiptera, Cicadellidae). The following new combinations are made: Atanus nitidus (Linnavuori, 1955) - comb. nov., Atanus cineratus (Linnavuori, 1959) - comb. nov., Atanus furcifer (Linnavuori, 1959) - comb. nov., Atanus lagunae (Linnavuori, 1959) - comb. nov., Atanus serricauda (Linnavuori, 1959) - comb. nov. Atanus joaquinus (Linnavuori, 1973) - comb. nov., Atanus rhopalus (Cheng, 1980)

- comb. nov., and Atanus trifurcatus (Cheng, 1980) - comb. nov.

KEYWORDS. Athysanini; Deltocephalinae; taxonomy.

RESUMO. Novas combinações em Atanus Oman (Hemiptera, Cicadellidae). As seguintes novas combinações são feitas: Atanus nitidus (Linnavuori, 1955) - comb. nov., Atanus cineratus (Linnavuori, 1959) - comb. nov., Atanus furcifer (Linnavuori, 1959) - comb. nov., Atanus lagunae (Linnavuori, 1959) - comb. nov., Atanus serricauda (Linnavuori, 1959) - comb. nov. Atanus joaquinus (Linnavuori, 1973) - comb. nov., Atanus rhopalus (Cheng, 1980) - comb. nov., e Atanus trifurcatus (Cheng, 1980) - comb. nov.

PALAVRAS-CHAVE. Athysanini; Deltocephalinae; taxonomia.
\end{abstract}

Menezes (1973) propôs o nome Linnatanus para substituir o homônimo junior Tubulanus Linnavuori, 1955, mas os autores subseqüentes ignoram esse ato nomenclatorial. Linnavuori \& DeLong (1976:36) sinonimizaram Tubulanus com Atanus Oman, 1938 sem, no entanto, formalizar as combinações das espécies. Posteriormente, Cheng (1980) descreveu duas novas espécies em Tubulanus. Finalmente, Kocak (1981) ignorando Menezes (1973) propôs um segundo nome para substituir Tubulanus.

O presente trabalho tem o objetivo de formalizar a transferência das espécies de Linnatanus (originalmente descritas em Tubulanus) e algumas espécies de Tubulanus para o gênero Atanus Oman, 1936.

\section{Atanus Oman, 1938}

Atanus Oman, 1938: 381. Espécie-tipo, Eutettix dentatus Osborn, 1923 (des. orig.).

Alanus DeLong \& Hersberger, 1947. Espécie-tipo, albidus DeLong \& Hersberger, 1947 (des. orig.).

Fulvanus Linnavuori, 1955: 110. Espécie-tipo, curvilinea Linnavuori, 1955 (des. orig.).

Tubulanus Linnavuori, 1955: 109. Espécie-tipo, nitidus Linnavuori, 1955 (des. orig.).

Linnatanus Menezes, 1973: 135 (= Tubulanus Linnavuori, 1955: 109, non Reinier, 1804).

Nerminia Kocak, 1981: 124 (= Tubulanus Linnavuori, 1955, non Reinier, 1804).
Atanus cineratus (Linnavuori, 1959) - comb. nov. Tubulanus cineratus Linnavuori, 1959: 305 Linnatanus cineratus; Menezes, 1973: 1325

Atanus furcifer (Linnavuori, 1959) - comb. nov. Tubulanus furcifer Linnavuori, 1959: 306 Linnatanus furcifer; Menezes, 1973: 135

Atanus joaquinus (Linnavuori, 1973) - comb. nov. Tubulanus joaquinus Linnavuori, 1973: 14-15

Atanus lagunae (Linnavuori, 1959) - comb. nov. Tubulanus lagunae Linnavuori, 1959: 304 Linnatanus lagunae; Menezes, 1973: 135

Atanus nitidus (Linnavuori, 1955) - comb. nov. Tubulanus nitidus Linnavuori, 1955: 110 Linnatanus nitidus; Menezes, 1973: 135

Atanus rhopalus (Cheng, 1980) - comb. nov. Tubulanus rhopalus Cheng, 1980: 106

Atanus serricauda (Linnavuori, 1959) - comb. nov. Tubulanus serricauda Linnavuori, 1959: 306 Linnatanus serricauda; Menezes, 1973: 135.

Atanus trifurcatus (Cheng, 1980) - comb. nov. Tubulanus trifurcatus Cheng, 1980: 108 


\section{REFERÊNCIAS}

Cheng, Y. J. 1980. New leafhopper taxa (Homoptera: Cicadellidae) from Paraguay. Journal of the Kansas Entomological Society 53: $61-118$

Linnavuori, R. 1955. Contributions to the Neotropical leafhopper fauna of the family Cicadellidae IV. Annales Entomologici Fennici 21: 100-129.
Linnavuori, R. 1973. Additional notes on the Cicadellidae fauna of Peru. Revista Peruana de Entomologia 16: 14-16.

Linnavuori, R. \& D. M. DeLong. 1976. New Neotropical leafhoppers from Peru and Bolivia (Homoptera: Cicadellidae). Revista Peruana de Entomologia 19: 29-38.

Menezes, M. de. 1973. Notas sobre Deltocephalinae da região Neotropical (Homoptera, Cicadellidae). Revista Brasileira de Entomologia 17: 131-136. 